\title{
Estimation de la prévalence de l'infection par le virus de l'hépatite C au Canada,
} 2011

\author{
Trubnikov $\mathbf{M}^{1^{*}}$, Yan $\mathrm{P}^{1}$, Archibald $\mathrm{C}^{1}$ \\ ${ }^{1}$ Centre de la lutte contre les maladies transmissibles et les infections, Agence de la santé publique du Canada, Ottawa (Ontario) \\ *Auteur-ressource : Maxim.Trubnikov@phac-aspc.gc.ca
}

\section{Résumé}

Contexte : Les estimations de la prévalence contribuent à notre compréhension de l'importance d'un état de santé particulier et à la planification d'interventions appropriées en matière de santé publique.

Objectif : Estimer la prévalence des infections chroniques par le virus de l'hépatite C (VHC), l'état de la séropositivité pour le VHC (anti-VHC) et la proportion d'infections par le VHC non diagnostiquées au Canada.

Méthodologie : On a utilisé une combinaison de la méthode de rétrocalcul et de la méthode basée sur un tableur. La méthode de rétrocalcul a permis d'estimer la prévalence de l'infection chronique par le VHC et la proportion de cas non diagnostiqués au moyen des données du Registre canadien du cancer sur un carcinome hépatocellulaire déclaré entre 1992 et 2008, ainsi que des données du Système canadien de surveillance des maladies à déclaration obligatoire sur les cas d'infection par le virus de l'hépatite C (VHC) déclarés entre 1991 et 2009 dans un modèle multistades de la progression de la maladie de Markov, dont les paramètres ont été ajustés pour le Canada. La méthode basée sur un tableur a divisé la population totale du Canada en sousensembles de population et a effectué des estimations de la taille de la population et de la prévalence de l'antiVHC pour chaque sous-ensemble. Les estimations de la taille des sous-populations ont été multipliées par les mesures de la prévalence de l'anti-VHC pour calculer la prévalence de l'anti-VHC par sous-population. Une mesure de l'élimination spontanée a été utilisée pour estimer le nombre de personnes atteintes d'une infection chronique par le VHC à partir des estimations du nombre de personnes séropositives pour le VHC.

Résultats : La méthode de rétrocalcul a permis d'estimer la prévalence de l'infection chronique par le VHC à $0,64 \%$ et la proportion de cas non diagnostiqués d'infection chronique par le VHC à $44 \%$ en 2011. La méthode basée sur un tableur a permis d'estimer la prévalence de l'anti-VHC à 0,96\% (intervalle plausible : de 0,61 \% à $1,34 \%)$ et de l'infection chronique par le VHC à 0,71 \% (0,45\%-0,99\%).

Interprétation : En combinant les estimations médianes des deux méthodes, on estime qu'entre 0,64 \% et $0,71 \%$ de l'ensemble de la population canadienne est atteinte d'une infection chronique par le VHC en 2011 et $44 \%$ de ces personnes n'ont pas reçu de diagnostic.

\section{Introduction}

L'infection chronique par le virus de l'hépatite $\mathrm{C}(\mathrm{VHC})$ touche environ $3 \%$ de la population mondiale (1). Environ trois personnes sur quatre touchées par une infection aiguë par le VHC n'éliminent pas spontanément le virus en six mois et développent une infection chronique par le VHC ainsi qu'un éventail de séquelles à long terme (2). Le diagnostic de l'infection par le VHC est habituellement fondé sur la détermination des anticorps du virus de l'hépatite $\mathrm{C}$ (anti-VHC) ou du matériel viral (p. ex. l'acide ribonucléique du VHC) (3) ainsi que sur certains tests enzymatiques de la fonction hépatique (4). Une séropositivité pour le VHC indique une infection passée ou actuelle par le VHC étant donné que les anticorps du VHC peuvent demeurer après l'élimination du virus. Une séropositivité au test d'ARN-VHC indique une infection actuelle, aiguë ou chronique, l'infection chronique par le VHC étant définie comme une séropositivité au test d'ARN-VHC pendant plus de six mois après la date présumée d'infection. 
Les estimations nationales de la prévalence contribuent à notre compréhension de l'importance d'un état de santé particulier et à la planification d'interventions appropriées en matière de santé publique (5). La prévalence des personnes séropositives pour le $\mathrm{VHC}$ au Canada était estimée à $0,78 \%$ de l'ensemble de la population canadienne en 2007, parmi lesquelles $21 \%$ étaient considérées comme non diagnostiquées à ce moment (6). Selon les données provenant des cycles 1 et 2 de l'Enquête canadienne sur les mesures de la santé (20072011), Rotermann et ses collègues estiment la séroprévalence des anticorps anti-VHC dans l'intervalle de $0,3 \%$ à $0,9 \%$ avec une médiane de $0,5 \%$. Environ $70 \%$ des personnes séropositives pour le $\mathrm{VHC}$ ont indiqué qu'elles n'avaient pas l'hépatite $C(7)$.

Toutefois, l'Enquête canadienne sur les mesures de la santé ne couvre pas les populations non résidentielles présentant un plus grand fardeau du VHC (8) (p. ex. les détenus, les sans-abri et les résidents des établissements de soins de santé) et, avec un taux de réponse juste au-dessus de $52 \%$ (7), l'Enquête peut avoir sous-échantillonné les populations résidentes fortement touchées par le VHC (p. ex. les utilisateurs de drogues injectables (UDI), les personnes atteintes d'une maladie chronique recevant un traitement d'hémodialyse et les immigrants qui ne parlaient ni anglais ni français). Par conséquent, l'analyse par Rotermann et ses collègues (7) a probablement sous-estimé la véritable séroprévalence de l'anti-VHC au Canada.

Étant donné le temps écoulé depuis la dernière détermination des estimations de la prévalence du VHC au Canada (6) et les limites possibles de l'analyse par Rotermann et ses collègues (7), cet examen visait à mettre à jour les estimations de la prévalence de l'infection chronique par le VHC, des personnes séropositives pour le VHC et de la proportion de cas non diagnostiqués d'infection chronique par le VHC au Canada.

\section{Méthodologie}

Les estimations de la prévalence de l'infection chronique par le VHC, des personnes séropositives pour le VHC et de la proportion de cas non diagnostiqués d'infection chronique par le VHC au Canada ont été élaborées à l'aide d'une combinaison de la méthode de rétrocalcul (9) et de la méthode basée sur un tableur (10).

Le rétrocalcul utilise l'occurrence observée d'événements subséquents pour faire des déductions au sujet de l'incidence des événements déclencheurs antérieurs qui les ont entraînés. Cette méthode a été récemment adoptée pour estimer l'incidence de l'infection par le VHC en France (11) et en Grande-Bretagne (12), où les données déclarées sur le carcinome hépatocellulaire associé au VHC et un modèle multistades de la progression de la maladie de Markov ont été utilisés pour rétrocalculer l'incidence historique du VHC. Nous avons utilisé une méthode de rétrocalcul avec les données du Registre canadien du cancer sur un carcinome hépatocellulaire signalé entre 1992 et 2008 et un modèle multistades de la progression de la maladie de Markov dont les paramètres ont été ajustés pour le Canada $(13,14)$ afin d'estimer la prévalence de l'infection chronique par le VHC et la proportion des infections par le VHC non diagnostiquées en 2011. La prévalence de l'hépatite C chronique pour 100 habitants a été estimée à l'aide des données stratifiées par cohorte de naissance de cinq ans selon la date de naissance. La prévalence globale a été estimée à l'aide du même modèle avec toutes les cohortes de naissance combinées. Un autre processus de rétrocalcul avec les données provenant du Système canadien de surveillance des maladies à déclaration obligatoire (SCSMDO) sur les cas d'infection par le VHC signalés entre 1991 et 2009 a été exécuté en parallèle afin de garantir la fiabilité des estimations de l'ancien. Comme les données enregistrées sur le VHC du SCSMDO n'étaient disponibles que pour six provinces et territoires canadiens qui représentent $88 \%$ de la population canadienne, les estimations fournies par le rétrocalcul ont été extrapolées à l'ensemble de la population canadienne.

Une méthode basée sur un tableur a été utilisée pour estimer le nombre de personnes séropositives pour le VHC indiquant une infection prévalente et non diagnostiquée au Canada en 2011. En utilisant cette méthode, les estimations de la taille de la population ont été multipliées par des mesures de la séroprévalence des anticorps anti-VHC (anti-VHC) pour produire des estimations de la prévalence des personnes séropositives pour le VHC. Une valeur de $26 \%$ a été utilisée pour décrire l'élimination spontanée du VHC par la population et pour estimer la prévalence de l'infection chronique par le VHC à partir d'une estimation des personnes séropositives pour le VHC (15). Ensuite, les estimations des personnes séropositives pour le VHC prévalentes ont été multipliées par la proportion de cas non diagnostiqués d'infection chronique par le VHC calculée à partir de la méthode de rétrocalcul pour produire le nombre de personnes potentiellement non diagnostiquées. 
L'ensemble de la population du Canada a été divisé en deux sous-ensembles de population et les estimations de la taille et de la prévalence de l'anti-VHC ont été élaborées pour chaque sous-groupe de la population. Les estimations de la taille de la population ont été élaborées à l'aide des données de documents publiés et d'un tableur personnalisé des données des cycles 1 et 2 issues de l'Enquête canadienne sur les mesures de la santé (Données non publiées. Agence de la santé publique du Canada, disponibles sur demande auprès de l'auteur). Le Tableau 1 présente les sources.

Les bases de données MEDLINE, EMBASE, GLOBAL HEALTH, SCOPUS et PROQUEST PUBLIC HEALTH ont été dépouillées afin de trouver des mesures de la prévalence de l'anti-VHC chez les populations d'intérêt au Canada et dans d'autres pays développés grâce à des documents pertinents publiés de 2000 à 2013 en anglais ou en français. Les bibliographies des études répertoriées ont également été dépouillées afin de trouver des articles pertinents en plus des ressources électroniques de Statistique Canada, Citoyenneté et Immigration Canada, Service correctionnel du Canada et l'Agence de la santé publique du Canada (ASPC) et Internet. Les demandes de renseignements ont été envoyées aux experts canadiens travaillant dans les domaines de la santé liée à la migration, aux études pénitentiaires, à l'abus d'alcool ou d'autres drogues, à la modélisation mathématique et à l'épidémiologie de l'hépatite $C$.

Au cours de l'examen, les mesures de la séroprévalence de l'anti-VHC ont été classées comme des " sousestimations ", des " surestimations » ou des " estimations appropriées » selon une évaluation subjective du degré auquel l'échantillon d'étude était représentatif de la population d'intérêt selon la description du plan d'étude dans la section sur les méthodes du document examiné. Les résultats de l'étude évalués comme des sousestimations ou des surestimations ont été utilisés pour déterminer les limites de l'intervalle plausible des estimations appropriées (10).

Un certain nombre de groupes de population ont été évalués comme ayant des études sur la prévalence de l'antiVHC de représentativité appropriée, y compris les personnes nées à l'étranger âgées de 14 et 79 ans, les utilisateurs actuels ou les anciens utilisateurs de drogues injectables, les sans-abri qui n'utilisent pas de drogues injectables, les détenus provinciaux et fédéraux et les résidents des établissements de soins de santé de longue durée. Pour ces groupes, les estimations de la prévalence du groupe d'études qui ont été classées comme étant des « estimations appropriées » ont été choisies si elles provenaient d'études de cohorte ou d'examens systématiques, ou, en leur absence, d'études comprenant une représentation géographique plus précise. Pour les personnes nées à l'étranger, on a utilisé un intervalle de mesures de prévalence de l'anti-VHC de 1,90\% (IC à $95 \%: 1,30-2,60$ ) (suggéré par Greenaway et ses collègues) (16). Pour les utilisateurs actuels de drogues injectables, y compris les personnes d'origine autochtone et les sans-abri qui utilisent des drogues injectables, on a utilisé un intervalle de séroprévalence de l'anti-VHC de 63 \%-69 \% (Données non publiées. I-Track: Surveillance améliorée des comportements à risque chez les utilisateurs de drogues injectables au Canada, phases 1-3. ASPC. 2013). Pour les anciens utilisateurs de drogues injectables, on a utilisé un intervalle de séroprévalence de l'anti-VHC de $28,5 \%$ (IC à $95 \%: 10,8-46,3$ ) issu d'un tableur personnalisé des données provenant des cycles 1 et 2 de l'Enquête canadienne sur les mesures de la santé (Données non publiées, ASPC, 2013). Pour les sans-abri qui n'utilisent pas de drogues injectables, on a utilisé un intervalle de mesures de la séroprévalence de l'anti-VHC de 0,8\% (données non publiées de la SAJR: Système de surveillance accrue des jeunes de la rue, phase 6 (2009-2011). ASPC, 2013) à 3,70\% (17). Pour les détenus des pénitenciers fédéraux, on a utilisé une estimation ponctuelle de la prévalence de l'anti-VHC à $24,0 \%$ fournie par le Service correctionnel du Canada pour 2011 (Données non publiées. Service correctionnel du Canada, 2013) et un intervalle de mesures d'une publication de De et ses collègues de $18,10 \%-37,10 \%$ (18). Pour les détenus des pénitenciers provinciaux, on a utilisé un intervalle de mesures de la séroprévalence de l'anti-VHC entre $18,5 \%$ (19) et $28,0 \%$ (20). Pour les résidents des établissements de soins de santé de longue durée, on a utilisé un intervalle de mesures de la séroprévalence de l'anti-VHC entre $1,4 \%(21)$ et $4,5 \%(20)$.

Pour le reste des groupes de population (y compris les Autochtones qui n'utilisent pas de drogues injectables et les personnes nées au Canada qui n'ont pas d'ascendance autochtone âgées de 0 à 13 ans et de 80 ans et plus qui n'utilisent pas de drogues injectables), les estimations médianes et les intervalles plausibles ont été déterminés à partir de données probantes indirectes sur les mesures de la prévalence de l'anti-VHC comparativement aux estimations de la prévalence de l'anti-VHC au sein de populations ayant des estimations fiables, comme les ratios de taux ou les valeurs inférieures ou supérieures aux taux de prévalence de l'anti-VHC 
mesurés chez les populations de comparaison. Par conséquent, la limite inférieure de l'estimation de la prévalence $(0,03 \%)$ pour les $14-44$ ans issue d'un tableur personnalisé des données provenant des cycles 1 et 2 de l'Enquête canadienne sur les mesures de la santé (Données non publiées. ASPC, 2013) a été utilisée comme la limite supérieure chez les enfants âgés de 0 à 13 ans, tandis que la limite inférieure a été établie à $0,01 \%$ et l'estimation médiane a été établie comme étant la moyenne des deux. Pour les résidents âgés de 80 ans et plus, la médiane et l'intervalle de prévalence chez les 14-44 ans issue d'un tableur personnalisé des données des cycles 1 et 2 provenant de l'Enquête canadienne sur les mesures de la santé (Données non publiées de l'ASPC, 2013) $(0,16 \%$ (de $0,03 \%$ à $0,29 \%$ ) ont été établis en tenant compte du fait qu'ils devraient être plus faibles que la prévalence chez les 45-79 ans (0,93\% (0,33 \%-1,53\%), mais plus élevés que dans le groupe d'âge de 013 ans $(0,02 \%(0,01 \%-0,03 \%)$ (comme le montre le tableur personnalisé des données provenant des cycles 1 et 2 de l'Enquête canadienne sur les mesures de la santé (Données non publiées. ASPC, 2013 ).

Pour les personnes nées au Canada qui n'ont pas d'ascendance autochtone née et qui n'utilisent pas de drogues injectables âgées de 14 et 79 ans, on a utilisé les mesures de la prévalence de l'anti-VHC issues d'un tableur personnalisé des données provenant des cycles 1 et 2 de l'Enquête canadienne sur les mesures de la santé (Données non publiées de l'ASPC, 2013) (0,20\% (IC à $95 \%$ : 0,10-0,30\%). Pour les Autochtones qui n'utilisent pas de drogues injectables, on a utilisé un multiple de 2,5 fois (coefficient trouvé dans l'étude de Uhanova et ses collègues (23)) le taux de séroprévalence issu d'un tableur personnalisé des données provenant des cycles 1 et 2 de l'Enquête canadienne sur les mesures de la santé (Données non publiées, ASPC, 2013) chez les personnes âgées de 14 et 79 ans nées au Canada qui n'ont pas d'ascendance autochtone et qui n'utilisent pas de drogues injectables. En raison des données très peu abondantes sur l'état de connaissance de la séropositivé pour le VHC, on a appliqué une estimation ponctuelle de cas non diagnostiqués d'infection chronique par le VHC issue de la méthode de rétrocalcul aux estimations ponctuelles des personnes atteintes d'une infection chronique par le VHC issues de la méthode basée sur un tableur et de la méthode de rétrocalcul pour calculer l'intervalle de personnes non diagnostiquées d'infection chronique par le VHC.

\section{Résultats}

La prévalence globale de l'infection chronique par le VHC (selon le rétrocalcul) a été estimée à $0,64 \%$ ou 220697 personnes en 2011. Au cours des 20 dernières années, la prévalence de l'infection chronique par le VCH à l'échelle nationale est passée de $0,6 \%$ à $0,7 \%$ (Figure 1). La plus forte prévalence d'infection chronique par le VHC a été observée dans la cohorte de naissance 1955-1959 (1,5\%), suivie par les cohortes de naissance 1950-1954 (1,25 \%), 1960-1964 (1,2\%), 1965-1969 (1,1\%) et 1970-1974 (0,8 \%). La prévalence de l'infection chronique par le VHC chez les personnes nées avant 1949 est passée d'environ $1 \%$ à une valeur inférieure au taux de prévalence globale au cours des 20 dernières années. La prévalence de l'infection chronique par le VHC chez les personnes nées après 1965 a augmenté, passant d'une valeur inférieure à une valeur supérieure au taux de prévalence globale. La prévalence de l'infection chronique par le VHC chez les personnes nées entre 1950 et 1964 est demeurée au-dessus de la prévalence globale tout au long des 20 dernières années. La méthode de rétrocalcul a également permis d'estimer que $44 \%$ des personnes atteintes d'une infection chronique par le VHC n'ont pas été diagnostiquées en 2011. 
Figure 1 : Estimation de la prévalence de l'infection chronique par le VHC ${ }^{1}$ (pour 100 habitants) au Canada, à partir d'un modèle de rétrocalcul ${ }^{2}$

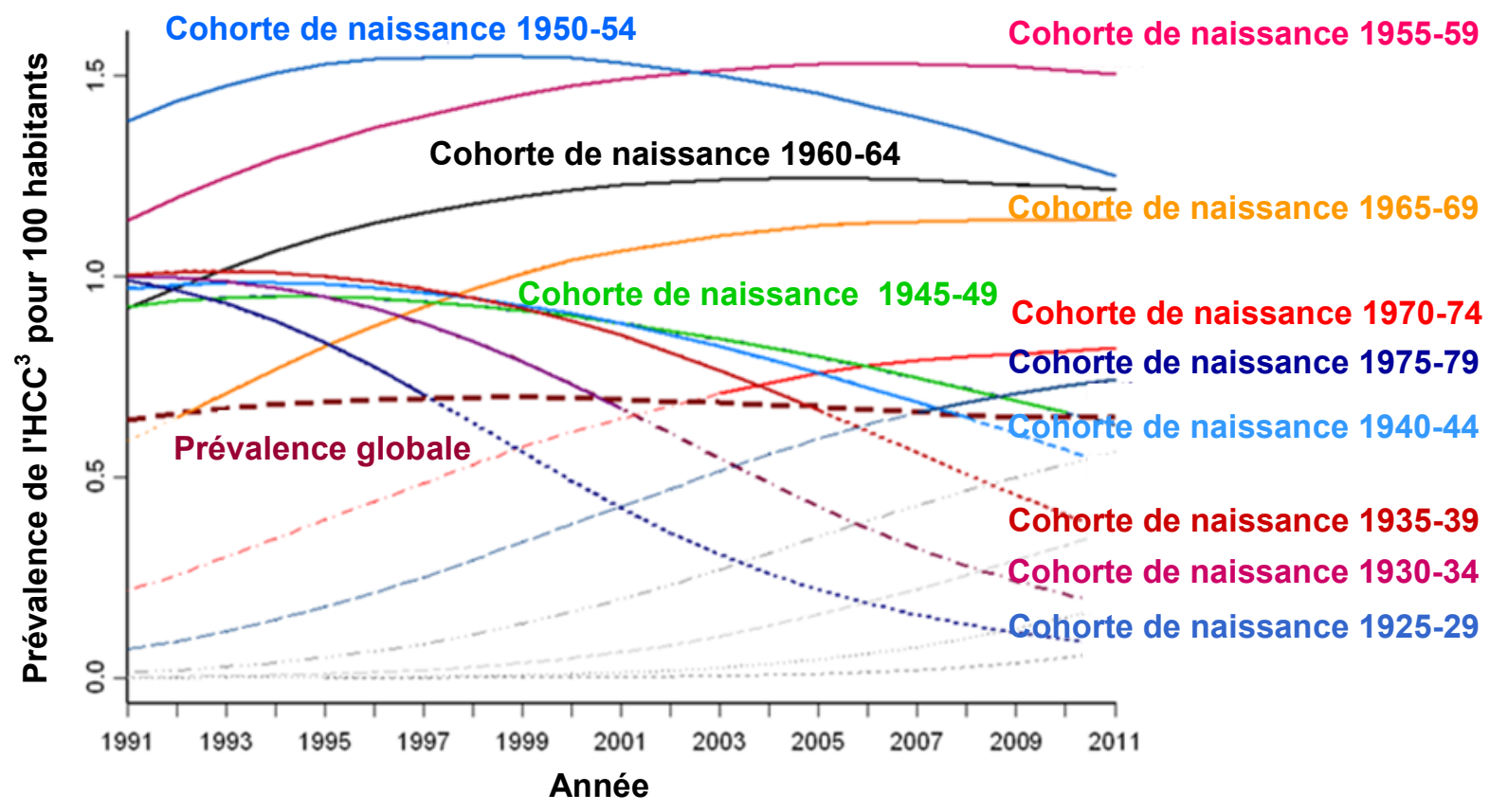

${ }^{1} \mathrm{VHC}=$ virus de l'hépatite $\mathrm{C}$

${ }^{2}$ Remarque : Les lignes pleines représentent la prévalence précise des cohortes de naissance lorsqu'elle était supérieure à la prévalence globale. Les lignes pointillées ont été utilisées lorsque la prévalence était inférieure à l'estimation globale.

${ }^{3} \mathrm{HCC}=$ Hépatite $\mathrm{C}$ chronique

La méthode basée sur un tableur a permis d'estimer la prévalence de l'anti-VHC au Canada en 2011 à 0,96 \% avec un intervalle de plausibilité de $0,61 \%$ à $1,34 \%$ (Tableau 1). Cet intervalle se traduit par une estimation de 332414 personnes (intervalle de plausibilité de : 210753 à 461517 personnes) séropositives pour le VHC en 2011 (Tableau 1). Après l'ajustement pour le taux d'élimination du VHC de $26 \%$, la méthode basée sur un tableur a permis d'estimer 0,71\% (intervalle de plausibilité de : 0,45 à 0,99\%) ou 245987 personnes (intervalle de plausibilité de : 155957 à 341522 personnes) qui n'avaient pas éliminé le virus et ont été considérés comme étant atteintes d'une infection chronique par le VHC en 2011.

Tableau 1 : Estimation de la prévalence et du nombre de personnes séropositives pour le VHC $^{1}$ ainsi que les intervalles de plausibilité associés, par population clé au Canada en 2011

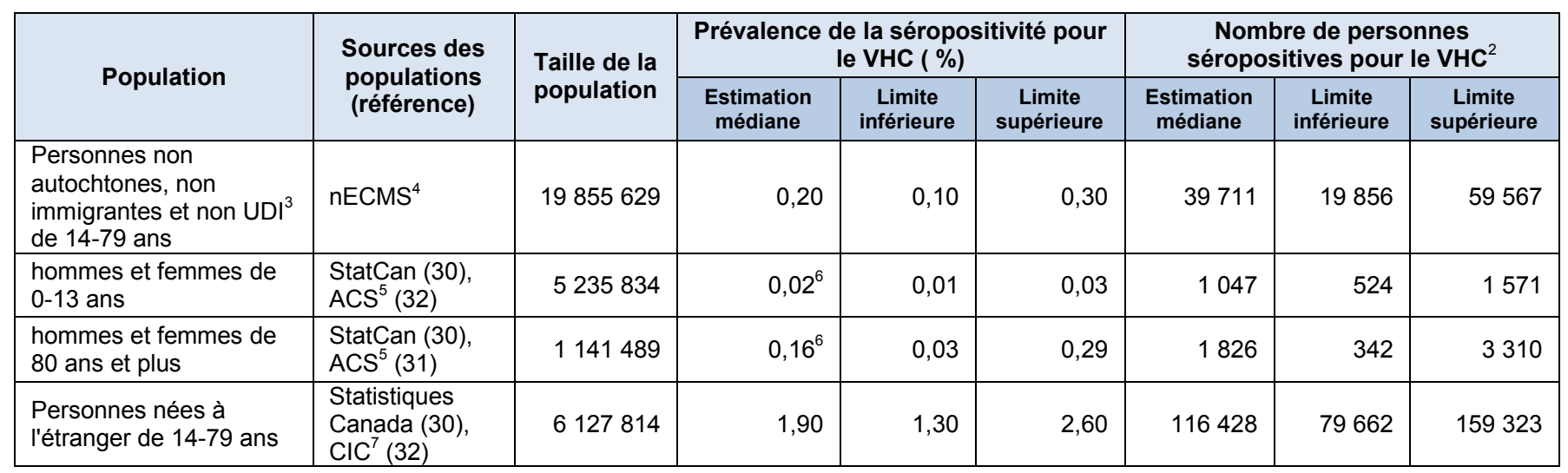




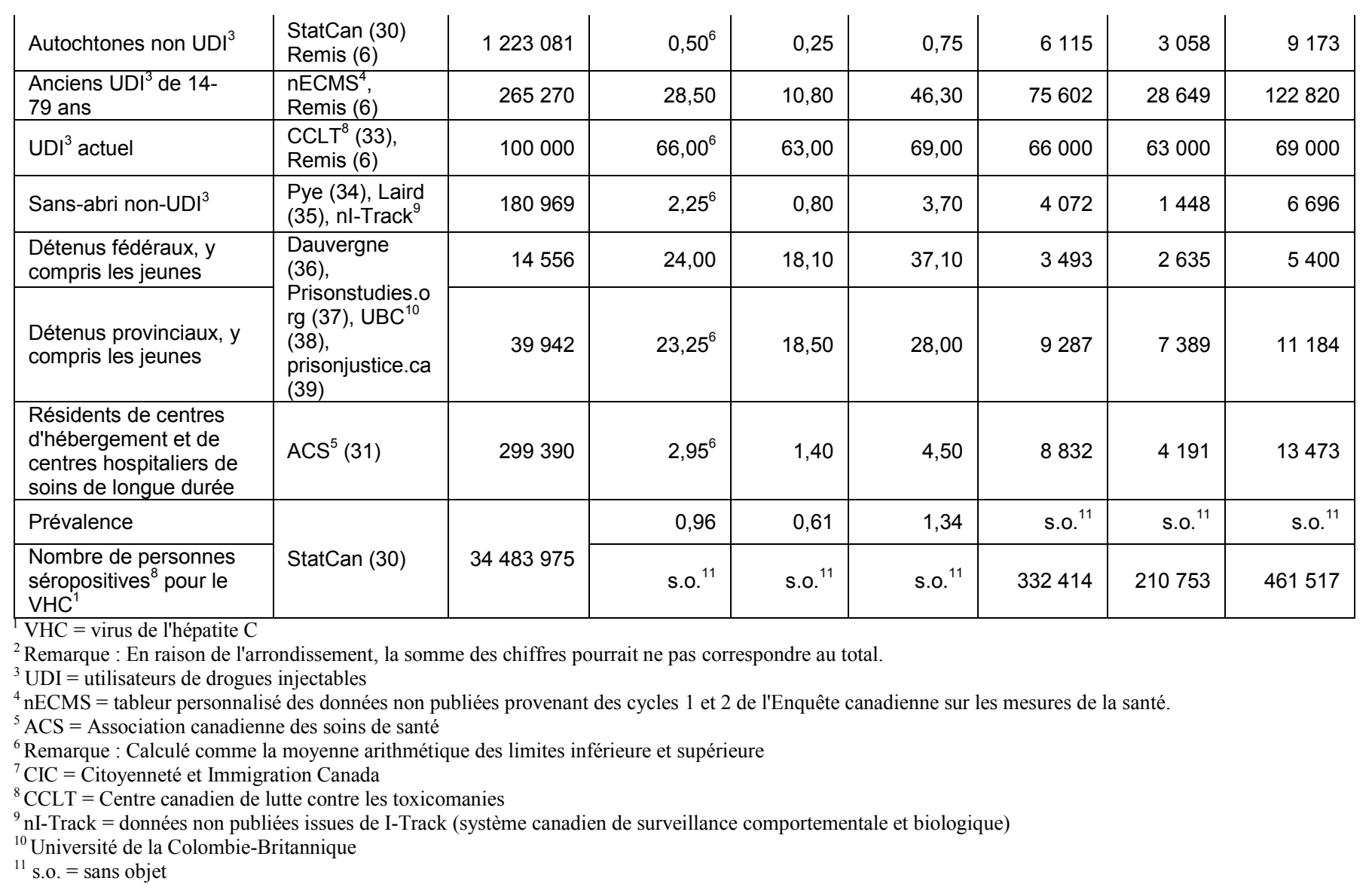

\section{Discussion}

En combinant les médianes des deux méthodes, entre $0,64 \%$ et $0,71 \%$ de l'ensemble de la population canadienne (de 220697 à 245987 personnes) était atteinte d'une infection chronique par le VHC au Canada en 2011. De ces personnes, $44 \%$ (de 97107 à 108234 personnes) n'avaient probablement reçu de diagnostic. Le nombre estimé de personnes séropositives pour le VHC était de 332414 personnes (environ $1 \%$ de la population canadienne) avec un intervalle de plausibilité de 210753 à 461517 personnes.

Les populations « cachées » comme les anciens utilisateurs et les utilisateurs actuels de drogues injectables ainsi que les sans-abri (environ $1 \%$ de la population canadienne totale) représentent près de $44 \%$ du nombre total de personnes séropositives pour le VHC. Les personnes nées à l'étranger représentent une autre proportion de 35 \% du nombre estimé de personnes séropositives pour le VHC au Canada en 2011.

Par comparaison avec une prévalence estimée à partir d'un exercice de modélisation par Remis (6), qui, comme la méthode de rétrocalcul, a utilisé un modèle multistades de la progression de la maladie de Markov, les estimations médianes de la prévalence de l'anti-VHC et de l'infection chronique par le VHC sont passées de $0,8 \%$ (6) à $1,0 \%$ et de $0,6 \%$ (6) à $0,7 \%$, respectivement. Cela indique que, entre 2007 et 2011 , les variations de la prévalence de l'anti-VHC et de l'infection chronique par le VHC (le cas échéant) sont survenues dans un intervalle étroit et que la majorité des personnes séropositives pour le VHC et celles atteintes d'une infection chronique par le VHC appartenaient à quelques populations clés au Canada.

Ces estimations de la prévalence sont comparables avec les estimations provenant d'une analyse des données recueillies aux États-Unis (24). En outre, l'estimation des populations cachées représentant $44 \%$ du nombre total 
de personnes séropositives pour le VHC est généralement comparable à l'estimation de 34 \% pour les populations comparables aux États-Unis (24).

D'autres résultats importants de cette analyse comprennent le fait que le groupe de naissance de 1950-1970 englobe actuellement la majeure partie de l'infection chronique par le VHC au Canada et que la nouvelle proportion estimée de personnes atteintes d'une infection chronique non diagnostiquée par le VHC en 2011 était de $44 \%$. Cette nouvelle estimation est environ deux fois plus élevée que l'estimation de Remis pour 2007 à $21 \%$ (6) et elle se situe entre les estimations des populations pour lesquelles on s'attend à observer des taux plus élevés de tests de dépistage du VHC, comme les utilisateurs de drogues injectables qui ne sont pas diagnostiqués à $20 \%-43 \%$ (Données non publiées. I-Track: Surveillance améliorée des comportements à risque chez les utilisateurs de drogues injectables au Canada, phases 1-3. ASPC. 2013) et les populations pour lesquelles on s'attend à observer de faibles taux de tests de dépistage du VHC, comme les patients hospitalisés non diagnostiqués à $56 \%(25)$ et l'estimation pondérée pour la population canadienne de 14-79 ans non diagnostiquée à $69,5 \%$ (7). L'estimation à $44 \%$ des personnes non diagnostiquées se situe également dans les intervalles de la proportion de cas non diagnostiqués au Canada chez les détenus (28-50\%) (26), les donneurs de sang pour la première fois (42-58 \% ne sont pas diagnostiqués) (27) et les hommes ayant des relations sexuelles avec d'autres hommes (44-75\% ne sont pas diagnostiqués) (Données non publiées de M-Track: Surveillance améliorée des comportements à risque chez les hommes ayant des relations sexuelles avec d'autres hommes, phases 1-2, ASPC, 2013). Elle est également comparable avec l'estimation des États-Unis de la proportion de personnes séropositives pour le VHC non diagnostiquées de 50,3 \% (28).

Les deux processus de rétrocalcul utilisés ensemble ont fourni l'occasion d'étalonner à l'interne les résultats du modèle afin d'améliorer la conformité aux données déclarées sur les cas d'infection par le VHC et les cas de carcinome hépatocellulaire. Les données du SCSMDO ont permis d'effectuer une estimation plus précise des récentes tendances, des jeunes cohortes de naissance et de l'ampleur globale de l'épidémie. Les données sur le carcinome hépatocellulaire ont permis d'adopter un modèle plus efficace des tendances historiques, des cohortes de naissance plus âgées et de la progression de la maladie, d'une manière similaire à celle utilisée par d'autres chercheurs dans le domaine (14). L'utilisation des deux ensembles de données dans le cadre d'un processus itératif a permis d'améliorer le modèle global et l'a rendu moins dépendant des limites d'un des ensembles de données. Nous avons également contrevalidé la prévalence annuelle du VHC prévue par le modèle par rapport à la prévalence issue de sources de données indépendantes, y compris les données de 2007 à 2011 de l'Enquête canadienne sur les mesures de la santé (7) et les données sur les cas déclarés d'infection par le VHC chez les patients issues de la base de données sur les congés des patients de l'Institut canadien d'information sur la santé (29). Même si les mesures absolues de la prévalence du VHC différaient des sources de données indiquées cidessus (possiblement en raison de la différence dans la méthodologie et dans la façon dont les résultats et la représentation géographique ont été définis), il y avait un consensus général dans la répartition des cas prévalents prévus/estimés du VHC par année de déclaration et par cohorte de naissance de même que pour les tendances temporelles.

Ces estimations peuvent être influencées par les données ainsi que par les limites méthodologiques comme la sous-déclaration des résultats, la combinaison des résultats des tests de dépistage des anticorps anti-VHC et d'ARN-VHC en une seule mesure de résultat et l'utilisation des données enregistrées de six provinces et territoires canadiens pour effectuer des inférences à propos de la prévalence du VHC dans l'ensemble du Canada. D'autres limites sont attribuables aux jugements de valeur et au choix des mesures de résultat pour des populations en particulier, à l'examen axé principalement sur la langue anglaise, à l'absence d'ajustement du modèle de rétrocalcul pour l'influence du traitement du VHC, ainsi qu'aux nombreuses hypothèses utilisées dans le processus d'estimation. Les méthodes utilisées pour élaborer des estimations de la prévalence du VHC décrites dans le présent document maximisent l'utilisation des données disponibles, sont fondées sur des sources de données indépendantes et, lorsqu'elles sont utilisées de façon conjointe et itérative, elles peuvent compenser les faiblesses individuelles. Néanmoins, nous prévoyons une modification des estimations de la prévalence au fur à mesure que de nouvelles données et des données améliorées sur la prévalence du VHC dans les populations canadiennes deviendront disponibles. 


\section{Remerciements}

Agence de la santé publique du Canada : Anton Andonov, Tory Atwood, Margaret Bodie, Lily Fang, Margaret Gale-Rowe, Ania Kemp, Salman Klar, Jane Njihia, Susanna Ogunnaike-Cooke, Dana Paquette, Lisa Smylie, Jill Tarasuk, Stephanie Totten, Tom Wong, Qiuying Yang. Santé Canada : Gregory Springer, Diego Garcia, Kathleen Lydon-Hassan. Service correctionnel du Canada : Jonathan Smith. Citoyenneté et Immigration Canada : Danielle Grondin, Dominique Massenat. Milieu universitaire canadien : Jordan Feld, Chris Greenaway, Naveed Janjua, Marina Klein, Mel Krajden, Robert Myers, Robert Remis, Morris Sherman, Rosie Thein, Julia Uhanova.

\section{Conflit d'intérêts}

Aucun

\section{Financement}

Ce travail a été appuyé par l'Agence de la santé publique du Canada.

\section{Références}

(1) Mohd Hanafiah K, Groeger J, Flaxman AD, Wiersma ST. Global epidemiology of Hepatitis C virus infection: New estimates of age-specific antibody to HCV seroprevalence. Hepatology. 2013; 57(4):1333-42.

(2) Agence de la santé publique du Canada. Foire aux questions sur l'hépatite C http://www.phac-aspc.gc.ca/hepc/faqfra.php

(3) Scott JD, Gretch DR. Molecular diagnostics of Hepatitis C virus infection: A systematic review. JAMA. 2007; 297(7):72432.

(4) Shapshak P, Somboonwit C, Drumright LN, et al. Molecular and contextual markers of Hepatitis C virus and drug abuse. Mol Diagn Ther. 2009; 13(3):153-79.

(5) Giesecke J. Modern infectious disease epidemiology. Arnold, Hodder Headline Group; 2001.

(6) Remis RS. Modélisation de l'incidence et de la prévalence de l'hépatite C et de ses séquelles au Canada, 2007. Rapport final. Agence de la santé publique du Canada, 2009. http://www.phac-aspc.gc.ca/sti-its-surv-epi/model/pdf/model07fra.pdf

(7) Rotermann M, Langlois K, Andonov A, Trubnikov M. Seroprevalence of Hepatitis B and C Virus Infections: Results from the 2007 to 2009 and 2009 to 2011 Canadian Health Measures Survey (CHMS). Health Reports. 2013; 24(11): 3-13.

(8) Edlin BR. Perspective: Test and treat this silent killer. Nature. 2011; 474:S18-19.

(9) Brookmeyer R, Gail MH. A method for obtaining short-term projections and lower bounds on the size of the AIDS epidemic. J Am Stat Assoc. 1988; 83: 301-8.

(10) Lyerta R, Gouws E, Garcia-Calleja JM et al. The 2005 workbook: An improved tool for estimating HIV prevalence in countries with low level and concentrated epidemics. Sex Transm Infect. 2006; 82:41-4.

(11) Beuffic-Burban S, Mathurin P, Valleron AJ. Modelling the past, current and future HCV burden in France: Detailed analysis and perspectives. Statistical Methods in Medical Research. 2009; 18:233-252.

(12) Sweeting MJ, De Angelis D, Brant LJ, Harris HE, Mann AG, Ramsey, ME. The burden of Hepatitis C in England. Journal of Viral Hepatitis. 2007; 14: 570-6.

(13) Thein HH, Yi Q, Dore GJ, Krahn MD. Estimation of stage-specific fibrosis progression rates in chronic Hepatitis C virus infection: a meta-analysis and meta-regression. Hepatology. 2008; 48, No. 2.

(14) Thein $\mathrm{HH}$, Yi Q, Krahn MD. Estimation of progression of Canadians infected with the Hepatitis $\mathrm{C}$ virus through the blood supply, 1986-1990, fourth revision of Hepatitis C prognostic model incorporating data from the compensation claimant report. Toronto: University of Toronto; 2011.

(15) Micallef JM, Kaldor JM, Dore GJ. Spontaneous viral clearance following acute Hepatitis C infection: A systematic review of longitudinal studies. J Viral Hepatitis. 2006; 13: 34-41.

(16) Greenaway C, Ma A, Klein M, et al. Seroprevalence of chronic Hepatitis C virus infection in immigrants and refugees: A systematic review and meta-analysis. Prepared for publication, 2013. In: Klein M. Screening for HCV in immigrant populations. Presentation at the PHAC HCV Screening and Burden Workshop, October 7-8, 2013, Ottawa, ON. 
(17) Moses S, Mestery K, Kaita KDE, Minuk GY. Viral Hepatitis in a Canadian street-involved population. Can J Public Health. 2002; 93:123-8.

(18) De P, Connor N, Bouchard F, Sutherland D. HIV and Hepatitis C virus testing and seropositivity rates in Canadian federal penitentiaries: A critical opportunity for care and prevention. Can J Infect Dis Med Microbiol. 2004; 15(4): 221-5.

(19) Poulin C, Alary M, Lambert G, et al. Prevalence of HIV and Hepatitis C virus infections among inmates of Quebec provincial prisons. CMAJ. 2007; 177(3):252-6.

(20) Prefontaine RG, Chaudhary RK, Mathias RG. Analysis of risk factors associated with Hepatitis B and C infection in correctional institutions in British Columbia. Can J Infect Dis. 1994; 5(4):153-6.

(21) Simor AE, Gordon M, Bishai FR. Prevalence of Hepatitis B surface antigen, Hepatitis C antibody, and HIV-1 antibody among residents of a long-term-care facility. J Am Geriatr Soc. 1992; Mar;40(3):218-20.

(22) Chien NT, Dundoo G, Horani MH, et al. Seroprevalence of viral Hepatitis in an older nursing home population. J Am Geriatr Soc. 1999; Sep;47(9):1110-3.

(23) Uhanova J, Tate RB, Tataryn DJ, Minuk GY. The epidemiology of Hepatitis C in Canadian indigenous population. Can J Gastroenterol. 2013; 27 (6):336-40.

(24) Chak E, Talal AH, Sherman KE, et al. Hepatitis C virus infection in USA: An estimate of true prevalence. Liver Int. 2011; 31(8):1090-101

(25) Houston S, Rowe BH, Mashinter L, et al. Sentinel surveillance of HIV and Hepatitis C virus in two urban emergency departments. Can J Emerg Med. 2004; 6(2):89-96.

(26) Jafari S, Copes R, Baharlou S, et al. Tattooing and the risk of transmission of Hepatitis C: A systematic review and metaanalysis. Int J Infect Dis. 2010; Nov;14(11):e928-40.

(27) Bowker SL, Smith LJ, Rosychuk RJ, Preiksaitis JK. A review of general Hepatitis C virus lookbacks in Canada. Vox Sanguinis. 2004; 86:21-7.

(28) Denniston MM, Klevens RM, McQuillan GM, Jiles RB. Awareness of infection, knowledge of Hepatitis C, and medical follow-up among individuals testing positive for Hepatitis C: National health and nutrition examination survey 2001-2008. Hepatology. 2012; 55:1652-61.

(29) Schanzer D, Paquette D, Lix L. Historical trends and projected hospital admissions for chronic hepatitis $C$ infection in Canada: A birth cohort analysis. Canadian Medical Association Journal Open. 2(3):E139-E144.

(30) Statistique Canada, Division de la démographie. Estimations de la population 0-100 +, 1971-2012. Estimations postcensitaires mises à jour, Juillet 2012.

(31) Association canadienne des soins de santé. Nouvelle direction pour les soins de longue durée en établissement. Tableau 2.http://www.cha.ca/wp-content/uploads/2012/11/CHA_LTC_9-22-09

(32) Citoyenneté et Immigration Canada. Faits et chiffres 2012 - Aperçu de l'immigration http://www.cic.gc.ca/francais/ressources/statistiques/faits2012/permanents/01.asp

(33) Canadian Centre on Substance Abuse. Injections Drug Use Overview. CCSA, 2013. http://www.ccsa.ca/Eng/Topics/Populations/IDU/Pages/InjectionDrugUsersOverview.aspx.

(34) Pye S. The Homeless Individuals and Families Information System (HIFIS) Initiative: Using information and communication technologies to build knowledge and understanding on homelessness, National Secretariat on Homelessness, Gatineau, Quebec, n.d., p. 1.

(35) Laird G. Shelter - Homelessness in a Growth Economy: Canada's 21st Century Paradox, Sheldon Chumir Foundation for Ethics in Leadership, Calgary, Alberta, 2007, p. 4.

(36) Dauvergne M. Statistiques sur les services correctionnels pour les adultes au Canada, Graphic 1. Comptes moyens des adultes sous surveillance dans la collectivité et en détention, Canada, 2010-2011 http://www.statcan.gc.ca/pub/85-002x/2012001/article/11715-fra.html.

(37) Federally incarcerated youth $12-17$ represent $4.9 \%$ of the total number of federal inmates. Canada. http://prisonstudies.org/country/canada.

(38) General Information on Canadian Prisons http://ccphe.familymed.ubc.ca/resources/general-information-on-canadian-prisons/

(39) Proportion of female inmates among provincial inmates. http://www.prisonjustice.ca/politics/facts_stats.html 